\title{
The Performance of Metagenomic Next-Generation Sequencing In Suspected Central Nervous System Infection
}

\author{
Li Liu \\ Hunan Provincial People's Hospital

\section{Yamin Yu} \\ Ningxiang People's Hospital

\section{Yi Shi} \\ Medical School of Nanjing University \\ Xin Su ( $\square$ suxinjs@163.com ) \\ Southern Medical University
}

\section{Research}

Keywords: mNGS, central nervous system infection, standards.

Posted Date: October 28th, 2021

DOl: https://doi.org/10.21203/rs.3.rs-1008030/v1

License: (c) (1) This work is licensed under a Creative Commons Attribution 4.0 International License. Read Full License 


\section{Abstract}

Background: To explore the performance of metagenomic next-generation sequencing (mNGS) technology in patients of suspected central nervous system infection.

Methods: From January 2018 to March 2021, 75 cases were enrolled in this retrospective analysis at Hunan Provincial People's Hospital. The clinical data of patients with suspected central nervous system infection who underwent cerebrospinal fluid mNGS were analyzed. The performances of mNGS were compared with the conventional methods.

Result: The sensitivity of mNGS, culture and smear in the diagnosis of 75 patients were $55 \%, 4.4 \%, 6.7 \%$; theirs'specificity were $54.3 \%, 100 \%, 100 \%$; theirs' positive predictive value (PPV ) were $57.9 \%, 100 \%, 100 \%$; theirs'negative predictive value (NPV) were $51.4 \%, 41.4 \%, 41.7 \%$, respectively. There was $41(54.6 \%)$ cases whose mNGS results were consistent with the final diagnosis. 22(29.3\%) mNGS results were considered as both mNGS positive/Case consistent; 19(25.3\%) mNGS results were considered as both mNGS negative/Case consistent; $18(24 \%)$ mNGS results were considered as both mNGS positive/Case inconsistent; $16(21.3 \%)$ mNGS results were considered as both mNGS negative/Case inconsistent. mNGS identified 35 irrelavant pathogens in this study.

Conclusion: mNGS showed a high sensitivity compared to conventional methods. There are still several challenges in clinical application. It is necessary to establish unified and effective standards for interpreting mNGS results.

\section{Introduction}

More than 100 known pathogens can cause central nervous system infection[1]. Viruses, bacteria, fungi, parasites, and amoebae are all the common pathogens[2]. Central nervous system (CNS) infection is a serious neurologic condition, but the etiology remains unknown in more than half patients [3]. So accurate etiology diagnosis is crucial for the successful treatment of central nervous system infection.

Conventional diagnostic test methods for CNS infections include cerebrospinal fuid (CSF) cell count, glucose, and protein measurements, CSF Gram staining, culture, biomarkers such as procalcitonin (PCT), creative reactive protein (CRP), serology and PCR detection. However, in the pathogens detection of CSF infection, traditional microbiological techniques such as culture, staining often have low sensitivity and PCR detection is also limited to the gene sequence of known pathogenic microorganisms[4]. mNGS, is a promising technique, offers a relatively unprejudiced diagnostic tool for all pathogens included in the database library in a single test, regardless of prior suspicions of candidate pathogens and without the need for isolation and culture[5]. As a novel diagnostic tool, mNGS has been used wildly for the identification of various pathogens from clinical samples such as tissues, CSF or plasma[6]. mNGS shows significant advantages in pathogen detection in meningitis [7], pulmonary infection [8, 9], sepsis [10], and other types of diseases[11-14]. However, numerous challenges remain in the interpretation of mNGS results when applying mNGS test into clinical microbiology laboratory diagnosis [15].

In CNS infection, multiple studies have demonstrated the ability of mNGS for the detection of viruses, bacteria, fungi, parasites, and some uncommon pathogens from CSF or brain tissue, indicating its ability to identify pathogens in CNS infection of unknown etiology[16-20]. Our study further demonstrated the overall ability of mNGS in the rapid diagnosis of CNS infection caused by bacteria, viruses, fungi, and M. tuberculosis.

\section{Methods}




\subsection{Study design and participants}

The cases of patients with suspected central nervous system infection, who were admitted to Hunan Provincial People's Hospital from January 1, 2018 to March 31, 2021, were reviewed. Each registered patient whose CSF were sent for mNGS was enrolled. The final diagnosis was adjudicated by a panel discussion following hospital discharge when the results of all tests and patients' responses to the antimicrobial therapy were available.

Meanwhile, clinical data of all enrolled patients, including CSF cell count, glucose, and protein measurements, CSF Gram staining, CSF culture, biomarkers such as white blood cell, procalcitonin (PCT), creative reactive protein (CRP), serology and PCR detection were collected.

\subsection{Procedure}

\subsubsection{CSF mNGS}

CSF sample was collected by standard procedures. And then sent to BGI-Huada Genomics Institute (Wuhan, China) for pathogens detection. Cerebrospinal fuid of $0.5 \mathrm{ml}$ were mixed and shaken with glass beads, and the DNA was extracted according to the steps of the TIANamp Micro DNA Kit (DP316; Tiangen Biotech, Beijing, China). The extracted DNA was broken ultrasonically into 200-300 bp fragments. An Agilent 2100 Bioanalyzer was used for quality control of the library and a Qubit dsDNA HS Assay Kit (Thermo Fisher Scientific) was used for quality control of the DNA library concentration. Low-quality and short (length<35bp) reads were removed. Then the remaining sequence were aligned to the current virus, bacterial, fungal, and protozoan databases (NCBI; ftp://ftp.ncbi. nlm.nih.gov/genomes), which consists of whole genome sequences of 4061 viral taxa, 2473 bacterial genomes or scaffolds, and genomic sequences for 199 fungi related to human infection and 135 parasites associated with human diseases.

For bacteria (excluding mycobacteria), viruses, fungi, and parasites, whose read number was in the top 10 in the complete belonging list, mNGS identified a microbe(on species level) [21]. The result was considered MTB-positive when at least one specific read was mapped to the species or genus level[22].

\subsection{Statistical analysis}

Sensitivity, specificity, positive predictive value, and negative predictive value of mNGS for the diagnosis of central nervous system infection were determined. Statistical analyses were performed using SPSS version 20.0 (IBM Corporation, Armonk, NY, USA). A p value less than 0.05 was considered statistically significant.

\section{Results}

\subsection{Population characteristics}

Between January 2018 and March 2021, in total, 75 patients who did mNGS, were enrolled, including 18 patients with viral encephalitis and or meningitis, 9 patients with tuberculosis meningitis, 15 patients with bacterial meningitis, 3 patients with fungal meningitis, and 30 patients with non-infectious CNS diseases. Non-infectious CNS conditions included autoimmune encephalitis $(7,23.3 \%)$, malignant tumor $(4,13.3 \%)$, secondary headache $(6,20 \%)$, epilepsy (4, $13.3 \%)$ and other non-infectious diseases $(9,30 \%)$.

The demographic and clinical characteristics of 75 patients with infectious diseases and non-infectious diseases are summarized in Table 1. In the infectious diseases group, the CSF protein was higher than non-infectious diseases 
group ( $1027 \pm 165.6$ vs $369.6 \pm 126.8, P=0.004)$. The $C S F /$ serum glucose ratio is significantly lower than non-infectious diseases group $(0.42 \pm 0.03$ vs $0.59 \pm 0.04, P=0.0009)$. While CSF WBC, chlorine, $L D H$ and CSF pressure showed no significant differences between two groups.

Overall, metagenomics sequencing showed satisfying pathogen detection rate compared to conventional methods. Culture reported 2 positive (one cryptococcal meningitis, one bacterial meningitis, smear reported 3 positive (one tuberculosis meningitis, two cryptococcal meningitis ), and mNGS detected 40 positive detection rate of mNGS was significantly higher than that of culture and smear in etiology detection.

The sensitivity of mNGS, culture and smear in the diagnosis of 75 patients were $55 \%, 4.4 \%, 6.7 \%$; theirs'specificity were $54.3 \%, 100 \%, 100 \%$; theirs'PPV were $57.9 \%, 100 \%, 100 \%$; theirs'NPV were $51.4 \%, 41.4 \%$,

$41.7 \%$, respectively(Table2). When we compared the consistency between the mNGS result and the final diagnosis. There was $41(54.6 \%)$ cases whose mNGS results were consistent with the final diagnosis. The remained cases's mNGS result were different from the final diagnosis(Figure 1).

22(29.3\%) mNGS results were considered as both mNGS positive/Case consistent including detection of 3 tuberculosis meningitis patients, 8 viral meningitis patients, 9 bacterial meningitis, 2 fungal meningitis. 19(25.3\%) mNGS results were considered as both mNGS negative/Case consistent including 5 autoimmune encephalitis, 3 malignant tumor, 4 headache, 3 epilepsy, and 4 other non-infectious diseases.

$18(24 \%)$ mNGS results were considered as both mNGS positive/Case inconsistent including 5 tuberculosis meningitis patients, 2 viral meningitis patients, 1 bacterial meningitis patient and 7 non-infectious diseases. It is worth mentioning that, within the 18 discrepancy cases, mNGS results were completely different from final diagnosis.

We explored the "false-positive" results of mNGS against final diagnosis. In total, mNGS

identified 35 irrelevant pathogens, including Acinetobacter baumannii(n=2), Alternaria alternaria 3( $n=2)$, Penicillium citri $(n=1)$, Paraphylococcus mutans $(n=1)$, Acinetobacter joenei $(n=1)$, Streptococcus oralis $(n=1)$, Rhodopyces cerevisiae $(n=1)$, Staphylococcus Koch $(n=1)$, Staphylococcus equis $(n=1)$, Candida albicans $(n=3)$, Human Herpesvirus type $4 \llbracket n=6)$, Cladosporium $(n=1)$, Nocardia nova $(n=1)$, Defective hypotrophic bacteria $(n=1)$, Forsyneria $(n=1)$, Actinomycetes chlamydia $(n=1)$, Human herpesvirus type $1(H S V 1)(n=1)$, Aspergillus chevalier $(n=1)$, Streptococcus pyogenes $(n=1)$, Pseudomonas aeruginosa $(n=1)$, Stenotrophomonas maltophilia $(n=1)$, Human herpesvirus $\beta 7(n=1)$, Human $\beta$ herpesvirus type 6A ( $n=1)$, Paraphitic Havnia $(n=1)$, Ornithyl-solubilizing Rauria $(n=1)$, Citrobacter workman $(n=1)$, Serratia Rubens $(n=1)$, Ophilia $(n=1)$, Klebsiella pneumoniae $(n=1)$, Leptovirus type 19 $(n=1)$, Leptovirus $(n=1)$, Human pegivirus $\ n=1)$, Hepatitis GB virus type $C(n=1)$, Aspergillus Niger $(n=1)$ and Aureomycin botrytis $(n=1)$. All of them were considered contamination, background microorganisms, and the detection of circulating cell-free DNA from non-pathogenic microbes. This result indicates that in diagnosis of CNS infection, mNGS may report some clinically irrelevant pathogens. So mNGS result must be discreetly interpreted considering clinical findings and other conventional tests.

16(21.3\%) mNGS results were considered as both mNGS negative/Case inconsistent including 1 tuberculosis meningitis patient, 8 viral meningitis patients, 5 bacterial meningitis patient and 1 fungal meningitis patient.

In mNGS subgroup, mNGS positive/Case consistent group had a lower CSF/serum glucose ratio than mNGS positive/Case inconsistent. while CSF WBC, protein and chlorine showed no significant differences among different groups (Table 3). 


\section{Discussion}

We retrospectively enrolled patients who suspected central nervous system infection during the period from January 2018 to March 2021, and attempted to analyze the performance of metagenomic next-generation sequencing. In this study, cerebrospinal fluid were tested by both mNGS and conventional methods. The positive rate of mNGS of $55 \%$ was significantly higher than that of conventional methods. It is similar with the $27.9-60 \%$ that has been reported in the literature[1]. mNGS showed a high sensitivity compared to conventional methods. But the specificity, positive predictive value, and negative predictive value of mNGS were lower. The NPV of mNGS when compared with the final diagnosis was $51.4 \%$. This does indicate that a negative mNGS result cannot exclude CNS infection. Therefore, clinical evaluation remains important when mNGS results are negative.

Pneumoniae N. meningitidis, H. influenzae, and L. monocytogenes are the most common pathogens of communityacquired suppurative meningitis [23]. It is different from previous study. There were 15 bacterial meningitis in our observation. The pathogens detected by mNGS were L. monocytogenes $(n=1)$, Streptococcus pneumoniae $(n=2)$, Wound pseudoescherichia( $n=1)$, Acinetobacter johnsonii $(n=1) \varangle K$ Klebsiella pneumoniae $₫ n=1)$, Streptococcus agalactiae $(n=1)$, Staphylococcus xylose $(n=1)$, Clostridium nucleate $(n=1)$. Among the 18 patients with virus encephalitis and/or meningitis, all pathogens identified via mNGS were DNA viruses, human herpes viruses (HSV1, EBV, and HHV-7) were the major pathogens, we normally lack diagnostic tests for these viruses[24], it is consistent with other findings[25, 26].

Of note is that the specificity of mNGS in the diagnosis of tuberculous meningitis is $100 \%$, which allows a negative mNGS test to be used as one of the diagnostic methods to exclude tuberculous meningitis. However, there was one case who were detected by smear but missed by mNGS in present study.

Despite mNGS has many advantages in detection pathogens, there are several challenges that might hinder its application in a clinical setting. At first, although mNGS provides microbial information that is difficult to obtain by traditional methods. However, the criteria cannot definitively determine which microbes are pathogenic because of the presence of contamination, background microorganisms, and the detection of circulating cell-free DNA from nonpathogenic microbes. It is necessary to establish unified and effective standards for interpreting the results.

The second challenge of $\mathrm{mNGS}$ is that the interpretation of $\mathrm{mNGS}$ results be rather confusing presently. It is unable to discriminate the pathogenicity status of the pathogens detected. Large amounts of information could be confusing and even misleading to physicians while making clinical decisions[27]. The third challenge is that samples may easily contaminated by environmental bacteria or human parasitic bacteria during sampling or sequencing, it is difficult to decide which pathogen is involved in infection, colonization, or contamination. Thus the results may interfere with the clinician's judgment. We believe that mNGS reports need to be interpreted very carefully, we have to interpreted it in combination with clinical findings and all laboratory tests, preferably in a multidisciplinary manner $[20,28]$. Only those microorganisms consistent with the patient's clinical symptoms will be considered pathogens. Finally, the cost-effectiveness of the broad application of mNGS also needs further investigation[29].

Our study has some limitations. First, this was a retrospective study. As a retrospective study, limited data and data accumulation were not controlled by the researcher. It enrolled small cases. In further studies, we will enlarge the sample size to validate this diagnostic potential in prospective cohort study. Second, we used preliminary data for analysis but still lack of control group simultaneously in our cohort. Besides, the mNGS results and identification may be easily influenced by many factors. At last, lack of a standard comparator for diagnostics, and classification bias were also the limitations of this study. 
In conclusion, mNGS had a superior advantage in detecting potential pathogens than conventional methods in suspected central nervous system infection. There are several challenges in clinical application for mNGS. It needs establishing unified and effective standards for interpreting mNGS results.

\section{Declarations}

\section{Acknowledgement}

None.

Authors' contributions: LL and YY collected the clinical data and processed statistical data, and LL drafted and edited the manuscript. XS and YS designed and revised the manuscript.

Funding: This work was supported by the Project of Natural Science Foundation of China (82070011), the Key Project of Jiangsu Commission of Health (K2019004), and the "333 project” of Jiangsu Province (BRA2019339), Changsha Municipal Natural ScienceFoundation[grant no. Kq2014193].

\section{Availability of data and materials}

The datasets during and/or analysed during the current study are available from the corresponding author on reasonable request.

\section{Ethics approval and consent to participate}

All procedures performed in this study involving human participants were conducted in accordance with the ethical standards of the Ethics Committee of The Hunan provincial People's Hospital, the 1964 Helsinki declaration and its later amendments, and with comparable ethical standards.

\section{Consent for publication}

Informed consent was waived since the nature of retrospective study. None of the data could be traced back to an identifiable patient.

\section{Competing interests}

All authors declare no conflict of interest.

\section{References}

1. Granerod J, Tam CC, Crowcroft NS, Davies NW, Borchert M, Thomas SL: Challenge of the unknown. A systematic review of acute encephalitis in non-outbreak situations. Neurology 2010, 75(10):924-932.

2. Erdem G, Kaptsan I, Sharma H, Kumar A, Aylward SC, Kapoor A, Shimamura M: Cerebrospinal Fluid Analysis for Viruses by Metagenomic Next-Generation Sequencing in Pediatric Encephalitis: Not Yet Ready for Prime Time? Journal of child neurology 2021, 36(5):350-356.

3. Anh NT, Nhu LNT, Hong NTT, Phuc TM, Tam PTT, Huong DT, Anh TT, Deng X, Nghia HDT, Nguyen TT et al: Viral Metagenomic Analysis of Cerebrospinal Fluid from Patients with Acute Central Nervous System Infections of Unknown Origin, Vietnam. Emerging infectious diseases 2021, 27(1):205-213. 
4. Lee AS, Lim IH, Tang LL, Wong SY: High frequency of mutations in the rpoB gene in rifampin-resistant clinical isolates of Mycobacterium tuberculosis from Singapore. Journal of clinical microbiology 2005, 43(4):2026-2027.

5. Sun W, Lu Z, Yan L: Clinical efficacy of metagenomic next-generation sequencing for rapid detection of Mycobacterium tuberculosis in smear-negative extrapulmonary specimens in a high tuberculosis burden area. International journal of infectious diseases : IJID : official publication of the International Society for Infectious Diseases 2021, 103:91-96.

6. Zhang XX, Guo LY, Liu LL, Shen A, Feng WY, Huang WH, Hu HL, Hu B, Guo X, Chen TM et al: The diagnostic value of metagenomic next-generation sequencing for identifying Streptococcus pneumoniae in paediatric bacterial meningitis. BMC infectious diseases 2019, 19(1):495.

7. Xing XW, Zhang JT, Ma YB, He MW, Yao GE, Wang W, Qi XK, Chen XY, Wu L, Wang XL et al: Metagenomic NextGeneration Sequencing for Diagnosis of Infectious Encephalitis and Meningitis: A Large, Prospective Case Series of 213 Patients. Frontiers in cellular and infection microbiology 2020, 10:88.

8. Wang J, Han Y, Feng J: Metagenomic next-generation sequencing for mixed pulmonary infection diagnosis. $B M C$ pulmonary medicine 2019, 19(1):252.

9. Chen Y, Feng W, Ye K, Guo L, Xia H, Guan Y, Chai L, Shi W, Zhai C, Wang J et al: Application of Metagenomic NextGeneration Sequencing in the Diagnosis of Pulmonary Infectious Pathogens From Bronchoalveolar Lavage Samples. Frontiers in cellular and infection microbiology 2021, 11:541092.

10. Greninger AL, Naccache SN: Metagenomics to Assist in the Diagnosis of Bloodstream Infection. The journal of applied laboratory medicine 2019, 3(4):643-653.

11. Thoendel MJ, Jeraldo PR, Greenwood-Quaintance KE, Yao JZ, Chia N, Hanssen AD, Abdel MP, Patel R: Identification of Prosthetic Joint Infection Pathogens Using a Shotgun Metagenomics Approach. Clinical infectious diseases : an official publication of the Infectious Diseases Society of America 2018, 67(9):1333-1338.

12. Ramesh A, Nakielny S, Hsu J, Kyohere M, Byaruhanga O, de Bourcy C, Egger R, Dimitrov B, Juan YF, Sheu J et al: Metagenomic next-generation sequencing of samples from pediatric febrile illness in Tororo, Uganda. PloS one 2019, 14(6):e0218318.

13. Mai NTH, Phu NH, Nhu LNT, Hong NTT, Hanh NHH, Nguyet LA, Phuong TM, McBride A, Ha DQ, Nghia HDT et al: Central Nervous System Infection Diagnosis by Next-Generation Sequencing: A Glimpse Into the Future? Open forum infectious diseases 2017, 4(2): ofx046.

14. Huang ZD, Zhang ZJ, Yang B, Li WB, Zhang CJ, Fang XY, Zhang CF, Zhang WM, Lin JH: Pathogenic Detection by Metagenomic Next-Generation Sequencing in Osteoarticular Infections. Frontiers in cellular and infection microbiology 2020, 10:471.

15. Piantadosi A, Mukerji SS, Ye S, Leone MJ, Freimark LM, Park D, Adams G, Lemieux J, Kanjilal S, Solomon IH et al: Enhanced Virus Detection and Metagenomic Sequencing in Patients with Meningitis and Encephalitis. mBio 2021, 12(4):e0114321.

16. Ge M, Gan M, Yan K, Xiao F, Yang L, Wu B, Xiao M, Ba Y, Zhang R, Wang J et al: Combining Metagenomic Sequencing With Whole Exome Sequencing to Optimize Clinical Strategies in Neonates With a Suspected Central 
Nervous System Infection. Frontiers in cellular and infection microbiology 2021, 11:671109.

17. Haston JC, Rostad CA, Jerris RC, Milla SS, McCracken C, Pratt C, Wiley M, Prieto K, Palacios G, Shane AL et al: Prospective Cohort Study of Next-Generation Sequencing as a Diagnostic Modality for Unexplained Encephalitis in Children. Journal of the Pediatric Infectious Diseases Society 2020, 9(3):326-333.

18. Hu Z, Weng X, Xu C, Lin Y, Cheng C, Wei H, Chen W: Metagenomic next-generation sequencing as a diagnostic tool for toxoplasmic encephalitis. Annals of clinical microbiology and antimicrobials 2018, 17(1):45.

19. Duan H, Li X, Mei A, Li P, Liu Y, Li X, Li W, Wang C, Xie S: The diagnostic value of metagenomic next囚generation sequencing in infectious diseases. BMC infectious diseases 2021, 21(1):62.

20. Chen J, Zhang R, Liu L, Qi T, Wang Z, Song W, Tang Y, Sun J, Liu D, Lin Y et al: Clinical usefulness of metagenomic next-generation sequencing for the diagnosis of central nervous system infection in people living with HIV. International journal of infectious diseases : IJID : official publication of the International Society for Infectious Diseases 2021, 107:139-144.

21. Zhang Y, Cui P, Zhang HC, Wu HL, Ye MZ, Zhu YM, Ai JW, Zhang WH: Clinical application and evaluation of metagenomic next-generation sequencing in suspected adult central nervous system infection. Journal of translational medicine 2020, 18(1):199.

22. Simner PJ, Buckwalter SP, Uhl JR, Wengenack NL: Identification of Mycobacterium species and Mycobacterium tuberculosis complex resistance determinants by use of PCR-electrospray ionization mass spectrometry. Journal of clinical microbiology 2013, 51(11):3492-3498.

23. Bijlsma MW, Brouwer MC, Bossuyt PM, Heymans MW, van der Ende A, Tanck MW, van de Beek D: Risk scores for outcome in bacterial meningitis: Systematic review and external validation study. The Journal of infection 2016, 73(5):393-401.

24. Shen H, Shen D, Song H, Wu X, Xu C, Su G, Liu C, Zhang J: Clinical assessment of the utility of metagenomic nextgeneration sequencing in pediatric patients of hematology department. International journal of laboratory hematology 2021, 43(2):244-249.

25. Steiner F, Pavlovic J: Subcellular Localization of MxB Determines Its Antiviral Potential against Influenza A Virus. Journal of virology 2020, 94(22).

26. Tyler KL: Acute Viral Encephalitis. The New England journal of medicine 2018, 379(6):557-566.

27. Qian YY, Wang HY, Zhou Y, Zhang HC, Zhu YM, Zhou X, Ying Y, Cui P, Wu HL, Zhang WH et al: Improving Pulmonary Infection Diagnosis with Metagenomic Next Generation Sequencing. Frontiers in cellular and infection microbiology 2020, 10:567615.

28. Simner PJ, Miller S, Carroll KC: Understanding the Promises and Hurdles of Metagenomic Next-Generation Sequencing as a Diagnostic Tool for Infectious Diseases. Clinical infectious diseases : an official publication of the Infectious Diseases Society of America 2018, 66(5):778-788.

29. Thakur KT: Application of Pathogen Discovery/Metagenomic Sequencing in CNS HIV. Current HIV/AIDS reports 2020, 17(5):507-513. 


\section{Tables}

Table 1 Clinical characteristics of participants

\begin{tabular}{|llll|}
\hline $\mathbf{N}=\mathbf{7 5}$ & CNS infection (n=45) & Non-infection CNS diseases $(\mathrm{n}=30)$ & $\mathrm{p}$ value \\
\hline Age ,median(rang,years) & $41.3 \pm 3.3$ & $44.8 \pm 3.9$ & 0.497 \\
Female/male & $32 / 13$ & $19 / 11$ & \\
\hline Blood laboratory examination (median) & & & 0.371 \\
\hline WBC(×109/L) & $12.1 \pm 1.6$ & $10.0 \pm 1.6$ & 0.771 \\
C-reaction protein, mg/L & $37.2 \pm 12.3$ & $32.08 \pm 10.56$ & 0.386 \\
Procalcitonin, ng/mL & $4.2 \pm 2.9$ & $1.6 \pm 0.7$ & 0.196 \\
CSF WBC, ${ }^{106 / L ~(M e a n) ~}$ & $739 \pm 387.7$ & $144.1 \pm 64.4$ & 0.004 \\
CSF protein, mg/L (Mean) & $1027 \pm 165.6$ & $369.6 \pm 126.8$ & 0.0009 \\
CSF/serum glucose ratio (Mean ) & $0.42 \pm 0.03$ & $0.59 \pm 0.04$ & 0.1756 \\
CSF Cl, mmol/L (Mean) & $119.1 \pm 1.4$ & $122.3 \pm 2.0$ & 0.490 \\
CSF LDH, U/L (Mean) & $148.7 \pm 47.2$ & $282.4 \pm 218.3$ & 0.118 \\
CSF pressure, mmH2O (Mean) & $222.6 \pm 18.4$ & $174.5 \pm 24.3$ & \\
\hline
\end{tabular}

Abbreviations: WBC,white blood cell; LDH, lactate dehydrogenase; $\mathrm{Cl}$, chlorine.

Table 2 Performance of metagenomic next generation sequencing (mNGS) compared with conventional test in suspected central nervous system infection

\begin{tabular}{|lllll|}
\hline $\mathrm{N}=75$ & & & & \\
& Sensitivity & Specificity & PPV & NPV \\
mNGS & $55 \%$ & $54.3 \%$ & $57.9 \%$ & $51.4 \%$ \\
Culture & $4.4 \%$ & $100 \%$ & $100 \%$ & $41.4 \%$ \\
Smear & $6.7 \%$ & $100 \%$ & $100 \%$ & $41.7 \%$ \\
\hline
\end{tabular}

Abbreviations: PPV, positive predictive value; NPV, negative predictive value.

Table 3 The comparisons of CSF laboratory examinations across mNGS subgroups 


\begin{tabular}{|c|c|c|c|c|c|c|}
\hline$N=75$ & $\begin{array}{l}\text { mNGS } \\
\text { positive/Case } \\
\text { consistent (a) }\end{array}$ & $\begin{array}{l}\text { mNGS } \\
\text { positive/Case } \\
\text { inconsistent } \\
\text { (b) }\end{array}$ & $\begin{array}{l}\text { mNGS } \\
\text { negative/Case } \\
\text { inconsistent } \\
\text { (c) }\end{array}$ & $\begin{array}{l}\text { mNGS } \\
\text { negative/Case } \\
\text { consistent (d) }\end{array}$ & $\begin{array}{l}\text { p value } \\
\text { between } \\
\text { group a } \\
\text { and b }\end{array}$ & $\begin{array}{l}\text { p value } \\
\text { between } \\
\text { group } \\
\text { c and d }\end{array}$ \\
\hline \multirow{2}{*}{$\begin{array}{l}\text { Age } \\
\text {,median(rang,years) } \\
\text { Female/male }\end{array}$} & $50 \pm 4.6$ & $47.11 \pm 4.7$ & $35.3 \pm 4.7$ & $39.5 \pm 6.2$ & & \\
\hline & $8 / 14$ & $7 / 12$ & $5 / 13$ & $7 / 9$ & & \\
\hline \multicolumn{7}{|l|}{$\begin{array}{l}\text { Blood laboratory } \\
\text { examination }\end{array}$} \\
\hline WBC(×109 /L) & $10.2 \pm 1.5$ & $10.7 \pm 2.5$ & $15.8 \pm 3.5$ & $11.1 \pm 2.5$ & 0.858 & 0.286 \\
\hline \multirow{2}{*}{$\begin{array}{l}\text { C-reaction protein, } \\
\mathrm{mg} / \mathrm{L}\end{array}$} & $58.6 \pm 27.0$ & $20.8 \pm 5.9$ & $27.9 \pm 12.1$ & $43.7 \pm 18.5$ & 0.187 & 0.470 \\
\hline & $10.5 \pm 6.1$ & $0.05 \pm 0.009$ & $0.56 \pm 0.26$ & $1.6 \pm 0.98$ & 0.192 & 0.335 \\
\hline $\begin{array}{l}\text { Procalcitonin, } \\
\mathrm{ng} / \mathrm{mL}\end{array}$ & $667.8 \pm 438.2$ & $163.9 \pm 70.9$ & $1101 \pm 843.7$ & $174.9 \pm 104.1$ & 0.341 & 0.318 \\
\hline CSF WBC, ${ }^{*} 106 / L$ & $1205 \pm 257.8$ & $883.4 \pm 308.6$ & $840.9 \pm 289.2$ & $420.7 \pm 206$ & 0.426 & 0.238 \\
\hline CSF protein, mg/L & $0.42 \pm 0.05$ & $0.58 \pm 0.05$ & $0.42 \pm 0.05$ & $0.56 \pm 0.06$ & 0.032 & 0.351 \\
\hline $\begin{array}{l}\text { CSF/serum glucose } \\
\text { ratio }\end{array}$ & $118 \pm 2.2$ & $120.8 \pm 2.4$ & $119.3 \pm 2.4$ & $122.8 \pm 2.6$ & 0.405 & 0.08 \\
\hline CSF Cl, mmol/L & & & & & & \\
\hline
\end{tabular}

\section{Figures}

The consistent proportion of mNGS and final diagnosis

mNGS positive/Case consistent mNGS negative/Case consistent

- mNGS positive/Case inconsistent m mNGS negative/Case inconsistent

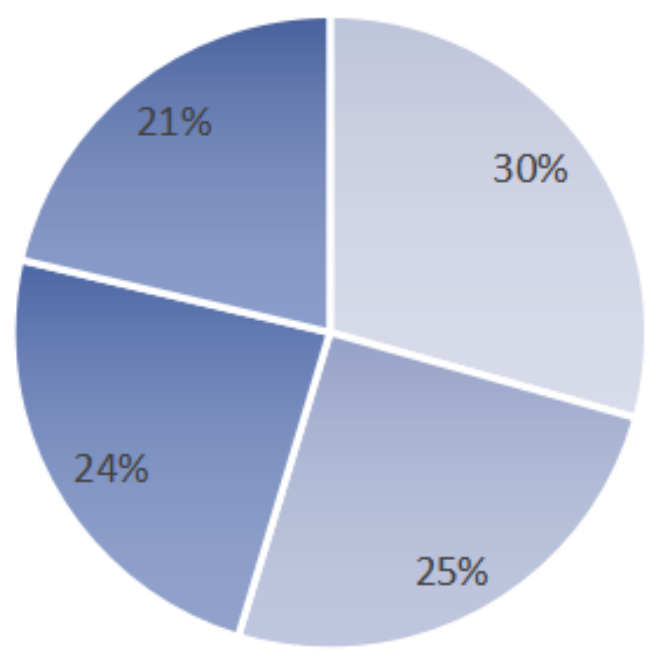


Figure 1

The consistent proportion of mNGS and final diagnosis 